\title{
Risk factors for dementia
}

\author{
Catriona D. McCullagh, David Craig, \\ Stephen P. Mcllroy \& A. Peter Passmore
}

There is little doubt that dementia is a very common cause of disability and dependency in our society. Since dementia of whatever type is usually more common with increasing age, then as population demographics change, so will the prevalence of dementia. Dementia is a generic term and the objective for clinicians, once dementia is suspected, is to attempt to define the cause. Alzheimer's disease is the most common cause of dementia, and in most centres vascular dementia would feature as the next most common aetiology. In some centres, Lewy body dementia is the second most common cause. Mixed Alzheimer's disease and vascular dementia would also feature high on the list at most centres.

This article will focus on risk factors for dementia and will concentrate only on Alzheimer's disease and vascular dementia. The risk factors for these will be discussed, and, since there have been significant advances in our understanding of the genetic component of Alzheimer's disease in particular, there is a separate section on genetic contribution.

\section{Environmental or non-genetic risk factors for Alzheimer's disease}

In contrast to the advances made in our understanding of genetic risk factors in Alzheimer's disease, identification of non-genetic or environmental risk factors has been slower. Non-inherited risk factors are likely to be important, as monozygotic twin concordance rates reach only $40 \%$. Different ethnic groups living in similar environments show comparable prevalence rates, again suggesting a role for environmental factors. Studies, which are often beset with methodological problems, have produced repeatedly conflicting results. Interpretation is further hampered by multiple interactions between different factors. Apolipoprotein E (ApoE) status in particular appears to modulate the influence of several environmental risk factors.

Age

With the exception of increasing age, none of the evidence for non-genetic risk factors is universally accepted. After 65 years of age, the incidence and prevalence of Alzheimer's disease doubles every 5 years. It is reasoned that older individuals have longer exposure to putative environmental and genetic influences. However, Alzheimer's disease in advanced age is not inevitable; differences in distribution and density of senile plaques and neurofibrillary tangles exist between patients and age-matched controls. Recent work suggests that the acceleration of incidence rates for Alzheimer's disease slows down in very old age (although there is no evidence of a rate decline), the corollary thus being that Alzheimer's disease is age-related rather than age-dependent (Gao et al, 1998).

\section{Gender and hormonal effects}

Even when controlling for differences in longevity, several studies have found that women are at increased risk for Alzheimer's disease. This is

Catriona McCullagh is a research fellow currently investigating genetic risk factors for stroke and dementia after stroke. David Craig is a lecturer and specialist registrar studying behavioural symptomatology in dementia. Stephen McIlroy is a geneticist and post-doctoral research fellow studying the genetic and molecular biology of Alzheimer's disease. Peter Passmore (Department of Geriatric Medicine, The Queen's University of Belfast, Whitla Medical Building, 97 Lisburn Road, Belfast BT9 7BL. Tel: 0289027 2153; fax: 0289032 5839; e-mail: p.passmore@qub.ac.uk) is research team leader involved in managing the Regional Centre for Cognitive Disorders, who has also published widely on various aspects of the molecular biology and genetics of Alzheimer's disease. 
complicated by the observation that men have a greater risk of developing vascular dementia, which may lessen the likelihood of developing pure Alzheimer's disease. Gender-related differences in risk could be at least partly ascribed to hormonal factors, as several studies suggest that oestrogen replacement can prevent or delay the onset of Alzheimer's disease. A 16-year follow-up of nearly 500 women found that hormone replacement therapy produced a 54\% reduction in risk of Alzheimer's disease (Kawas et al, 1997). However, a recent trial exploring the value of therapeutic oestrogen in subjects with Alzheimer's disease was unable to demonstrate any improvement in cognition or disease progression (Mulnard et al, 2000). Oestrogen may be implicated in Alzheimer's disease in several ways, for example, via reduction in $\beta$-amyloid deposition, improvement in cerebral blood flow, neuroprotection or suppression of ApoE.

\section{Oxidative and inflammatory stress}

Increased levels of oxidative stress are a biochemical feature of Alzheimer's disease. Trials of the antioxidants vitamin $\mathrm{E}$ and selegiline showed a delay in nursing home placement compared with patients receiving placebo. However, there was no effect on cognition (Sano et al, 1997). Oxidative changes may constitute a response rather than a cause. The same may apply to the described inflammatory changes within the Alzheimer brain. It is hypothesised that $\beta$-amyloid excites an immune response via microglial cell activation. Antiinflammatory drugs may inhibit this response and delay nerve cell damage. Several studies have reported that intake of non-steroidal anti-inflammatory drugs (NSAIDs) is negatively associated with the risk of developing Alzheimer's disease (Beard et $a l, 1998)$. As with all case-control studies, confounding bias may weaken interpretations. Alzheimer's disease patients may be less likely to receive NSAIDs because they are less able to complain of pain. In the central nervous system, cyclooxygenase-2 (COX-2) is present both in neurons and in reactive microglial cells. Therefore, COX-2 inhibition may favourably affect neuronal function as well as inflammation. Clinical trials involving selective COX-2 inhibitors in Alzheimer's disease are underway.

\section{Vascular risk factors}

Several case-control studies have reported an inverse relationship between smoking and risk of Alzheimer's disease. Studies based on prevalent patients may be flawed, as smoking may cause the death of subjects before they enter the age group where susceptibility to
Alzheimer's disease is more pronounced. Results from a pooled analysis of incident patients demonstrated increased risk of Alzheimer's disease in current and former smokers (Launer et al, 1999). Positive relationships between Alzheimer's disease and other vascular risk factors, including insulin-dependent diabetes, vascular disease, hypertension and electrocardiogram ischaemia, have also been reported (Stewart et al, 1999). Fish consumption, an important source of $\Omega-3$ polyunsaturated fatty acids, was inversely related to dementia, and in particular to Alzheimer's disease, in the Rotterdam incident analysis (Kalmijn et al, 1997).

Positive, negative and neutral associations have been noted between blood pressure levels and Alzheimer's disease, although a recent clinical trial has revealed the potential preventive effect of antihypertensive treatment on the incidence of dementia, especially of Alzheimer's disease (Forette et al, 1998).

\section{Head trauma}

The dementia pugilistica of boxers is associated with pathological changes similar to those of Alzheimer's disease. This led to the hypothesis that previous head injury increased the risk of subsequent Alzheimer's disease. Studies of head trauma have been hampered by recall bias, as relatives of affected patients may be more likely to remember previous events than those of healthy controls. A recent metaanalysis failed to implicate head trauma as a risk factor for Alzheimer's disease (Launer et al, 1999).

\section{Education}

Poor education has been cited as a risk factor for Alzheimer's disease, especially in males (Ott et al, 1999). Better education may reflect greater cognitive capacity and reserve, thus deferring the onset of the illness. Similar arguments apply to head size and dementia risk. It is unclear whether it is education obtained in childhood or the life-time acquisition of knowledge (thus implicating challenging occupations) that is protective. Assuming the latter, a trial of cognitive training in individuals at risk of dementia is currently running in the USA.

\section{Chemical exposure}

Solvents and heavy metals have been implicated as possible aetiological factors. In theory, high levels of iron could encourage free radical formation and oxidant stress. Current research into genetic polymorphisms within iron-handling genes may yield useful results. The involvement of both lead and solvents is generally discounted. 
The well-publicised relationship between Alzheimer's disease and aluminium is difficult to assess (McLachlan et al, 1996). Epidemiological studies were initiated in response to the observation of aluminium within plaques and tangles. Moreover, aluminium can cause abnormal phosphorylation of tau, a major component of the neurofibrillary tangle. Aluminium is ubiquitous and caution is needed when interpreting positive associations based on population exposure to high aluminium levels in drinking water. Studies concerning the role of aluminium compounds in antacids and antiperspirants have generally yielded negative results. As events at Camelford, England (where large quantities of aluminium sulphate were allowed to contaminate the water supply) indicate, aluminium is potentially neurotoxic (Altmann et al, 1999). It is much less clear whether aluminium is responsible for the chronic neurodegeneration of Alzheimer's disease. Only large-scale prospective analysis can help to resolve the issue.

\section{Depression}

The frequent coexistence of dementia and depression has stimulated speculation that associations exist between the two conditions. Prospective studies have again yielded opposing results. Depressive symptoms have been shown to increase the risk of subsequent cognitive decline (Yaffe et al, 1999). This has been tempered by research suggesting that depression is merely an early manifestation, rather than a predictor, of Alzheimer's disease (Chen et al, 1999). High levels of cortisol may be associated with depression, and indeed stress, and may also cause neuronal death (Lerner, 1999).

\section{Parental age}

Both old and young maternal ages have been found by different studies to increase the risk of subsequent Alzheimer's disease in offspring - the role of paternal age is even less clear (Rocca et al, 1991).

\section{Risk factors for vascular dementia}

Research into dementia has concentrated on Alzheimer's disease, and much progress has been made in revealing possible genetic and neuropathological mechanisms. However, over the past few years there has been renewed interest in vascular dementia, the only preventable type of dementia. This highlights the importance of identifying potential modifiable risk factors. Unlike Alzheimer's disease, which is primarily a cortical dementia, and progressive supranuclear palsy, Parkinson's disease and Huntington's disease, which are all subcortical, vascular dementia can be cortical (e.g. large cortical infarcts), subcortical (e.g. lacunar states, or Binswagers) or both cortical and subcortical.

The prevalence of dementia would appear to be constant worldwide, but the type of dementia varies from country to country. Alzheimer's disease is generally accepted as the most common type of dementia in the Western world, whereas vascular dementia is more common in Asian countries (Jorm et al, 1987).

Vascular dementia accounts for $24-48 \%$ of dementing illnesses in older people, with an incidence of 6-10 in 10000 per year in those aged over 70 years. Prevalence varies from $1.2 \%$ to $4.2 \%$ of those aged 65 years or above, increasing with age. There would appear to be a slight male preponderance. The average duration of the illness is 5 years and survival is less than that for Alzheimer's disease (Hebert \& Brayne, 1995).

Clinical diagnosis requires the presence of: (a) dementia; (b) cerebrovascular disease; and (c) a temporal relation between vascular disease and dementia.

There has been difficulty in establishing agreed criteria for diagnosis, definition and assessment of subjects, and much of the uncertainty stems from diagnostic issues. Furthermore, problems arise owing to the common co-occurrence of Alzheimer's disease and vascular dementia. To improve the sensitivity and specificity of the clinical diagnosis of vascular dementia, two sets of criteria have been developed over the past 5 years by the State of California Alzheimer's Disease Diagnostic and Treatment Centers (ADDTC) and the National Institute of Neurological Disorders and Stroke (NINDS), with support from the Association Internationale pour la Recherche et l'Enseignement en Neurosciences (AIREN). The NINDS-AIREN criteria, expressly developed for research purposes, have a high specificity, making them most useful tool in this area.

Vascular dementia designates cognitive deterioration caused by occlusion of cerebral arteries and loss of cerebral tissue and encompasses a heterogeneous group of conditions (Box 1 ).

\section{Demographic risk factors}

It is widely accepted that advancing age is a risk factor for stroke and thereby vascular dementia, as are male gender and race/ethnicity (Gorelick, 1997). 
Box 1 Conditions encountered in vascular dementia

1. Multi-infarct dementia Usually results from multiple, large cerebral infarcts arising from emboli of cardiogenic origin or the internal carotid arteries. It has a different course to, and is readily differentiated from, Alzheimer's disease. It accounts for only $27 \%$ of cases.

2. Strategic single-infarct dementia Primarily involves infarcts in one or more of: the thalamus, frontal, white matter, basal ganglia and angular gyrus (14\%).

3. Lacunar dementia Results from atherosclerotic and degenerative changes within the walls of the deep penetrating arterioles. Usually caused by hypertension and diabetes. It is the most common cause of vascular dementia $(30 \%)$.

4. Binswanger's subcortical arteriosclerotic encephalopathy At autopsy, white-matter lesions show partial loss of myelin sheaths and of oligodendroglial cells and axons, producing a decrease in the density of the white matter. There is severe stenosis or occlusion of the small vessels supplying white matter caused by fibrohyaline material.

5. Mixtures of 1, 2 and 3 Brought on by multiple occlusions of cerebral arteries, producing mixed cortical and subcortical dementias.

6. Haemorrhagic dementia Usually from uncontrolled hypertension/large arteriovenous malformations, subarachnoid haemorrhage and intracerebral haemorrhage with or without vasospasm.

7. Subcortical dementias Similar to 3, but caused by genetically determined arteriolopathies. These include cerebral autosomally dominant arteriopathy with subcortical infarcts and leukoencephalopathy, as well as familial amyloid angiopathies and coagulopathies.

8. Combined Alzheimer's disease Including Alzheimer's disease patients with cerebral infarcts or with haemorrhagic lesions resulting from amyloid angiopathy.

\section{Vascular risk factors}

The diagnosis of vascular dementia is associated with a history of cerebrovascular disorders, therefore some risk factors are the same as for stroke.

\section{Stroke}

Dementia after stroke is particularly associated with lacunar infarcts, left-hemispheric lesions and infarcts in the left-posterior and left-anterior cerebral territories (Tatemichi et al, 1993).

One year after stroke, the probability of new-onset dementia is $5.4 \%$ in patients over 60 years of age and $10.4 \%$ in patients over 90 years (Tatemichi et al, 1992). Four years after a first lacunar infarct, $23 \%$ of patients develop dementia, that is, 4-12 times more than controls (Loeb et al, 1992).

In one study, the prevalence of dementia after ischaemic stroke in patients aged 60 or over was $26.3 \%$, that is, 9.4-fold higher than that of a strokefree control group, after adjusting for age and level of education. This, however, included patients who were suffering from dementia prior to stroke. In this study, stroke was the underlying cause of dementia in $56.1 \%$, while in $36.4 \%$ it was presumed to be owing to the cumulative effects of stroke and Alzheimer's disease, as suggested by a pre-stroke history of functional impairment (Tatemichi et al, 1992).

The increased incidence of dementia after stroke suggests that cognitive decline is not only a direct consequence of the damage to the brain caused by stroke, but that there are additional processes related to the occurrence of stroke and the development of dementia. Approximately one-third of cases cannot be explained by the sequelae of stroke or hypoxic or ischaemic disorders.

\section{Hypertension}

Hypertension is the most important remediable risk factor for stroke (especially lacunar infarction) and vascular dementia (Lindsay et al, 1997). There is substantial evidence to suggest that elevated blood pressure earlier in life is a risk factor for dementia in later life. Late-life cognitive impairment can be associated with normal or low-normal blood pressure (Skoog et al, 1996). Thus, blood pressure may be an important early-life predictor of dementia, and control of blood pressure may prevent or delay 
dementia onset. Alternatively, mild systolic blood pressure increase in patients with vascular dementia could have beneficial effects (Meyer et al, 1986).

The Vascular Dementia arm of the Systolic Hypertension in Europe trial investigated whether antihypertensive drug treatment could reduce the incidence of dementia. The results showed that treatment was associated with a lower incidence of dementia. If 1000 hypertensive patients were treated with antihypertensive drugs for 5 years, 19 cases of dementia might be prevented (Forette et al, 1998). It may be that antihypertensive agents have actions other than blood-pressure lowering (e.g. endothelial arterial wall modification), which add to their ability to slow the progression of dementia or cognitive impairment. The Cochrane Library is conducting a review of effectiveness of antihypertensives in dementia.

\section{Diabetes}

Along with hyperlipidaemia, diabetes is associated with a reduction in cerebral perfusion due to microangiopathy, often resulting in lacunar infarctions (Desmond et al, 1993).

\section{Lipids}

Elevated levels of low-density lipoproteins, in particular, have been shown to be an independent risk factor for the development of dementia with stroke. However, no relationship has been found between lipid levels and the risk of probable Alzheimer's disease, suggesting that dyslipidaemia may be most relevant to the occurrence of dementia with a vascular component (Moroney et al, 1999).

\section{Smoking}

Smoking has been shown to be a risk factor by Meyer et al (1988), whereas the Canadian Study of Health and Aging (Lindsay et al, 1997) did not show any association, a possible explanation being that there could be a decreased survival of smokers.

\section{Cerebral white-matter lesions}

Cerebral white-matter lesions refer to a state of demyelination in the subcortical structures of the brain and arteriosclerotic changes of the small penetrating arteries and arterioles in the white matter. These are seen more frequently in vascular dementia than in other dementias or in normal, cognitively intact elderly subjects. White-matter changes are associated with risk factors for stroke and are themselves independent predictors of post-stroke dementia (Miyos et al, 1992).
Cerebral white-matter lesions on magnetic resonance imaging in association with the classic cardiovascular risk factors - stroke/myocardial infarction, factor VIIc, fibrinogen level and, in those over 65 years of age, hypertension and plasma cholesterol - have been associated with lower tests of cognitive function and significantly associated with lower scores on tests of subjective mental decline (Breteler et al, 1994).

In patients with first-ever lacunar infarction, mortality, stroke recurrence, risk of dementia and risk of dependence are significantly higher in patients with white-matter changes. Other vascular risk factors are history of myocardial infarction (Gorelick, 1997) and atrial fibrillation (Ott et al, 1997).

\section{Non-vascular risk factors}

Occupational exposure

Interestingly, the Canadian Study of Health and Aging (Lindsay et al, 1997) also showed an elevated odds ratio for vascular dementia in patients who had occupational exposure to pesticides and fertilisers (odds ratio 2.6) and liquid plastics or rubbers (odds ratio 2.59). These should be studied in more detail as, again, they are potentially preventable causes of vascular dementia.

Alcohol

Several studies have shown an increased risk of vascular dementia in patients with a history of alcohol misuse (Lindsay et al, 1997), but not all report this (Meyer et al, 1988). This warrants further study, as it is potentially preventable.

\section{Other non-vascular risk factors}

Psychological stress early in life (Peerson \& Skoog, 1996), poor formal education (Lindsay et al, 1997) and blue-collar occupation (Peerson \& Skoog, 1996) have been associated with a higher incidence of vascular dementia.

\section{Genetic factors}

Several rare genetic diseases have been associated with stroke and subsequent vascular dementia. These include autosomal dominant hereditary cerebral haemorrhage with amyloidosis - Dutch type and familial vascular encephalopathies, such as cerebral autosomal dominant arteriopathy with subcortical infarcts and leukoencephalopathy (CADASIL) (Haan et al, 1990).

Several studies (Lenzen et al, 1986; Gerdes, 1994) have shown an increased risk of stroke or coronary heart disease in patients with the ApoE 
polymorphism, a known genetic risk factor for Alzheimer's disease. This may suggest a shared pathogenic relationship and unifying explanation. Alzheimer's disease patients also have some degree of vascular changes and one-fifth have vascular lesions (Jellinger et al, 1990), which probably contributes to their cognitive decline. The link between stroke and Alzheimer's disease seems to be higher than that expected by chance and may reduce the period of preclinical Alzheimer's disease.

Furthermore, ApoE could influence the pathogenesis of dementia with stroke through its effects on lipid metabolism and atherosclerosis.

In summary, there are many risk factors for vascular dementia, most of which are shared in common with stroke. As vascular dementia is the only preventable type of dementia, it is important that these risk factors are identified early in life to reduce not only stroke risk, but risk of dementia.

\section{Genetic risk factors for dementia}

Alzheimer's disease is the most common form of dementia, and the fact that the vast majority of genetic risk investigations are concerned with Alzheimer's disease reflects this. In familial disease, risk to first-degree relatives has been estimated to range from $24 \%$ to over $50 \%$ at the age of 90 years. Concordance rates in monozygotic twins have been reported to be 40-50\% (Pericak-Vance \& Haines, 1995). Early-onset families show an autosomal dominant mode of inheritance with age-related penetrance, and mutations in one of at least three genes have been shown to confer this susceptibility. The three genes in question are those encoding the amyloid precursor protein (APP) gene on chromosome 21, presenilin 1 (PS-1) on chromosome 14 and presenilin 2 (PS-2) on chromosome 1. Late-onset families show a more complicated mode of inheritance, which, like sporadic disease, probably indicates the involvement of a number of genetic and other factors.

One stratagem for identifying genetic risk factors is to type deoxyribonucleic acid (DNA) polymorphisms in genes that may be involved in the pathogenesis of that disease. Polymorphisms in DNA are the existence of differences in the DNA sequence at a particular locus. These are more frequent than mutations and can be found in the DNA of both controls and disease subjects.

Although most reported genetic risk factors remain controversial, the ApoE $\varepsilon 4$ allele has been identified as a risk factor for Alzheimer's disease in most populations. Individuals who possess the $\varepsilon 4$ allele are approximately four times more likely to suffer from Alzheimer's disease than those without it, and this risk increases with $\varepsilon 4$ dose. ApoE $\varepsilon 4$ also reduces the age at which one can expect to develop the disease. However, possession of ApoE $\varepsilon 4$ is neither necessary nor sufficient for disease initiation, and this has prompted a search for other genetic factors that may influence risk for Alzheimer's disease.

An intronic polymorphism in the early-onset gene for PS-1 was reported to increase the risk of sporadic Alzheimer's disease, but this finding was not universally replicated. A form of vascular dementia, CADASIL, has been linked to the human Notch3 gene (Joutel et al, 1997). The gene product has been shown to interact with human PS-1, possibly a common pathway for neurodegeneration and dementia.

Other research groups have concentrated on receptors for ApoE, reasoning that if ApoE conferred increased risk then polymorphisms in ApoE receptors may modify that risk. A polymorphism in very low-density lipoprotein receptor (VLDL-R) was reported to increase risk in a Japanese Alzheimer's disease population (Okuizumi et al, 1995), and this was confirmed in a Scottish Alzheimer's disease population (Brookes et al, 1997), but other studies could not replicate this finding. Our group has reported an increased risk for Alzheimer's disease in Northern Ireland associated with a different genotype of VLDL-R from that originally reported by the Japanese group, reflecting the different population origins. Another ApoE receptor, the lowdensity lipoprotein receptor-related protein (LRP), was shown to increase risk for Alzheimer's disease in some studies. The identity of this molecule as a risk factor was further enhanced by the identification of a region of chromosome 12 near to the LRP locus as possibly being involved in familial Alzheimer's disease aetiology. However, other groups, including ours, failed to confirm this association.

Alpha-2 macroglobulin (A2M), a ligand for LRP and a serum pan-protease inhibitor, has also been shown in some studies to increase risk of Alzheimer's disease. It resides close to LRP on the short arm of chromosome 12 and is also in the region of the locus linked to familial Alzheimer's disease. As in the previous case, other groups, including our own, failed to replicate this finding. Polymorphisms in another protease inhibitor, $\alpha-1$ antichymotrypsin (AACT), have been linked with risk of Alzheimer's disease. This molecule, like ApoE, is a component of amyloid plaques, which increased its candidature as a risk factor. However, this also proved controversial.

Variations in the mitochondrial genome have been estimated to account for up to $20 \%$ of late-onset Alzheimer's disease cases. Polymorphisms in several genes, such as the COI and COII sub-units of the cytochrome $\mathrm{C}$ oxidase complex, have been associated with increased risk of Alzheimer's disease. Mutations in mitochondrial genes have also 
been implicated in the aetiology of Parkinson's disease. Point mutations in genes encoding subunits of NADH-ubiquinone oxoreductase have been proposed to lead to increased susceptibility to oxidative damage and neurodegeneration.

Parkinson's disease shares other susceptibility loci with Alzheimer's disease. One of these is the oestrogen receptor gene. This gene is a well-known, although not widely replicated, risk factor for Alzheimer's disease. Recently, a Japanese group reported that one allele of a polymorphism in this gene increased the risk of both Alzheimer's disease and Parkinson's disease (Isoe-Wada et al, 1999). Mutations in the $\alpha-$ synuclein gene are also thought to increase the risk of both diseases. Another gene that appears to be involved in the pathogenesis of both Parkinson's disease and Alzheimer's disease is the tau gene on chromosome 17. Mutations in this gene are now known to cause frontal temporal dementia with parkinsonism linked to chromosome 17 (FTDP-17). Clinically, this syndrome is very similar to Alzheimer's disease, although the frontal lobe abnormalities do not match the Alzheimer's disease phenotype. FTDP-17 has also been confused with Pick's disease.

In the search for genetic risk factors for both Alzheimer's disease and vascular dementia, generalised vascular disease genetic risk factors have also been examined. Recently, our group in collaboration with groups from London and Cardiff reported that the I allele of a common insertion (I) / deletion (D) polymorphism in the angiotensinconverting enzyme (ACE) was associated with increased risk of Alzheimer's disease (Kehoe et al, 1999). This finding has since been replicated in a US population, enhancing the candidacy of ACE as a risk factor for Alzheimer's disease. The results of studies on vascular dementia have not been as fruitful. Of the few reports in the literature, most are contradictory with no clear candidate risk factor for this disease identified so far. However, given the effect that molecular genetics has had on complex diseases, especially Alzheimer's disease, the identification of definitive genetic risk factors for other forms of dementia cannot be too far off.

\section{References}

Altmann, P., Cunningham, J., Dhanesha, U., et al (1999) Disturbance of cerebral function in people exposed to drinking water contaminated with aluminium sulphate: retrospective study of the Camelford water incident. British Medical Journal, 319, 807-811.

Beard, C. M., Waring, S. C., O'Brien, P. C., et al (1998) Nonsteroidal anti-inflammatory drug use and Alzheimer's disease: a case-control study in Rochester, Minnesota, 1980 through 1984. Mayo Clinic Proceedings, 73, 951-955

Breteler, M. M .B., van Swieten, J. C. \& Bots, M. L. (1994) Cerebral white matter lesions, vascular risk factors, and cognitive function in a population based study: The Rotterdam Study. Neurology, 44, 1246-1252.

Brookes, A. J., Howell, W. M., Woodburn, K., et al (1997) Presenilin-1, presenilin-2 and VLDL-R associations in early onset Alzheimer's disease. Lancet, 350, 336-337.

Chen, P., Ganguli, M., Mulsant, B. H., et al (1999) The temporal relationship between depressive symptoms and dementia: a community-based prospective study. Archives of General Psychiatry, 56, 261-266.

Desmond, D. W., Tatemichi, T. K., Paik, M., et al (1993) Risk factors for cerebrovascular disease as correlates of cognitive function in a stroke free cohort. Archives of Neurology, 50, 162-166.

Forette, F., Seux, M. L., Staessen, J. A., et al (1998) Prevention of dementia in randomised double-blind placebocontrolled Systolic Hypertension in Europe (Syst-Eur) trial. Lancet, 352, 1347-1351.

Gao, S., Hendrie, H. C., Hall, K. S, et al (1998) The relationships between age, sex, and the incidence of dementia and Alzheimer disease: a meta-analysis. Archives of General Psychiatry, 55, 809-815.

Gerdes, L. U. (1994) Genotype and cardiovascular disease: a quantitative overview of 42 studies. Genetic Epidemiology, 11, 294.

Gorelick, P. B. (1997) Status of risk factors for dementia associated with stroke. Stroke, 28, 459-463.

Haan, J., Lanser, J. B. K., Zijderveld, I., et al (1990) Dementia in hereditary cerebral haemorrhage with amyloidosisDutch type. Archives of Neurology, 47, 965-967.

Hebert, R. \& Brayne, C. (1995) Epidemiology of vascular dementia. Neuroepidemiology, 14, 240-257.

Isoe-Wada, K., Maeda, M., Yong, J., et al (1999) Positive association between an estrogen receptor gene polymorphism and Parkinson's disease with dementia. European Journal of Neurology, 4, 431-435.

Jellinger, K., Danielczyk, W., Fischer, P., et al (1990) Clinicopathological analysis of dementia disorders in the elderly. Journal of Neurological Sciences, 95, 239-258.

Jorm, A. F., Korten, A. E. \& Henderson, A. S. (1987) The prevalence of dementia: a quantitative integration of the literature. Acta Psychiatrica Scandinavica, 76, 465-479.

Joutel, A., Vahedi, K., Corpechot, C., et al (1997) Strong clustering and stereotyped nature of Notch 3 mutations in CADASIL patients. Lancet, 350, 1511-1515.

Kalmijn, S., Launer, L. J., Ott, A., et al (1997) Dietary fat intake and the risk of incident dementia in the Rotterdam Study. Annals of Neurology, 42, 776-782.

Kawas, C., Resnick, S., Morrison, A., et al (1997) A prospective study of estrogen replacement therapy and the risk of developing Alzheimer's disease: the Baltimore Longitudinal Study of Aging. Neurology, 48, 1517-1521.

Kehoe, P. G., Russ, C., Mcllroy, S., et al (1999) Variation in DCP1, encoding ACE, is associated with susceptibility to Alzheimer disease. Nature Genetics, 21, 71-72.

Launer, L. J., Andersen, K., Dewey, M. E., et al (1999) Rates and risk factors for dementia and Alzheimer's disease: results from EURODEM pooled analyses. EURODEM Incidence Research Group and Work Groups. European Studies of Dementia. Neurology, 52, 78-84.

Lenzen, H. J., Assman, G., Buchwalsky, R., et al (1986) Association of apolipoprotein E polymorphism, low density lipoprotein cholesterol and coronary artery disease. Clinical Chemistry, 32, 778-781.

Lerner, A. J. (1999) Alzheimer's disease in males: endocrine issues and prospects. Journal of Clinical Endocrinology and Metabolism, 84, 3416-3419.

Lindsay, J., Hebert, R. \& Rockwood, K. (1997) The Canadian Study of Health and Aging - Risk Factors for Vascular Dementia. Stroke, 28, 526-530.

Loeb, C., Gandolfo, C., Croce, R., et al (1992) Dementia associated with lacunar infarction. Stroke, 23, 1225-1229.

McLachlan, D. R., Bergeron, C., Smith, J. E., et al (1996) Risk for neuropathologically confirmed Alzheimer's disease and residual aluminum in municipal drinking water employing weighted residential histories. Neurology, $\mathbf{4 6}$ 401-405. 
Meyer, J. S., Judd, B. W., Tawakina, T., et al (1986) Improved cognition after control of risk factors for multi-infarct dementia. Journal of the American Medical Association, 256, 2203-2209.

—, McClintik, K., Rogers, R. L., et al (1988) Aetiological considerations and risk factors for multi-infarct dementia. Journal of Neurology and Neurosurgical Psychiatry, 51, 14891497.

Miyos, S., Takano, A., Teramoto, J., et al (1992) Leukoariosis in relation to prognosis for patients with lacunar infarction. Stroke, 233, 1434-1438

Moroney, J. T., Tang, M.-X., Bergkind, L., et al (1999) Low density lipoprotein cholesterol and the risk of dementia with stroke. Journal of the American Medical Association, 282, 254-260.

Mulnard, R. A., Cotman, C. W., Kawas, C., et al (2000) Estrogen replacement therapy for treatment of mild to moderate Alzheimer disease: a randomized controlled trial. Alzheimer's Disease Cooperative Study. Journal of the American Medical Association, 283, 1007-1015.

Okuizumi, K., Onodera, O., Namba, Y., et al (1995) Genetic association of the very low density lipoprotein (VLDL) receptor gene with sporadic Alzheimer's disease. Nature Genetics, 2, 207-209.

Ott, A., Breteler, M. M. B., de Bruyne M. C., et al (1997) Atrial fibrillation and dementia in a population-based study: the Rotterdam Study. Stroke, 28, 316-321.

-, van Rossum, C. T., van Harskamp, F., et al (1999) Education and the incidence of dementia in a large population-based study: the Rotterdam Study. Neurology, 52, 663-666.

Peerson, G. \& Skoog, I. (1996) A prospective population study of psychosocial risk factors for late-onset dementia. International Journal of Geriatric Psychiatry, 11, 15-22.

Pericak-Vance, M. A. \& Haines, J. L. (1995) Genetic susceptibility to Alzheimer disease. Trends in Genetics, 11, 504-508.

Rocca, W. A., van Duijn, C. M., Clayton, D., et al (1991) Maternal age and Alzheimer's disease: a collaborative reanalysis of case-control studies. EURODEM Risk Factors Research Group. International Journal of Epidemiology, 20 (suppl. 2), S21-S27.

Sano, M., Ernesto, C., Thomas, R. G., et al (1997) A controlled trial of selegiline, alpha-tocopherol, or both as treatment for Alzheimer's disease. The Alzheimer's Disease Cooperative Study. New England Journal of Medicine, 336, 1216-1222.

Skoog, I., Lernfelt, B., Landahl, S., et al (1996) 15-year longitudinal study of blood pressure and dementia. Lancet 347, 1141-1145.

Stewart, R., Prince, M. \& Mann, A. (1999) Vascular risk factors and Alzheimer's disease. Australian and New Zealand Journal of Psychiatry, 33, 809-813.

Tatemichi, T. K., Desmond, D. W., Mayeux, R., et al (1992) Dementia after stroke: baseline frequency, risks, and clinical features in a hospitalized cohort. Neurology, 42, 1185-1193.

_- Paik, M., et al (1993) Clinical determinants of dementia related to stroke. Annals of Neurology, 33, 568-575.

Yaffe, K., Blackwell, T., Gore, R., et al (1999) Depressive symptoms and cognitive decline in nondemented elderly women: a prospective study. Archives of General Psychiatry, 56, 425-430.

\section{Multiple choice questions}

1. The following have clearly been shown to be risk factors for Alzheimer's disease:

a female gender

b head injury

c aluminium

d age

e maternalage.
2. Regarding Alzheimer's disease:

a oestrogen therapy has recently been shown to improve cognition and disease progression

b vitamin $E$ has recently been shown to improve cognition

c insulin-dependent diabetes has been shown to be an independent risk factor

d treatment of isolated systolic hypertension has been found to reduce incidence

e non-steroidal anti-inflammatory drugs are assoicated with risk of Alzheimer's disease.

3. Regarding dementia:

a Alzheimer's disease is more common than vascular disease in Asian countries

b the survival rate in vascular dementia is lower than in Alzheimer's disease

c $20 \%$ of patients with Alzheimer's disease have cerebral vascular lesions

d more than $25 \%$ of patients may develop dementia following a stroke

e elevated blood pressure early in life is a risk factor for dementia.

4. Gene mutations on the following chromosomes have been shown to be associated with earlyonset of familial Alzheimer's disease:

a chromosome 1

b chromosome 19

c chromosome 12

d chromosome 21

e chromosome 14.

5. ApoE $\varepsilon 4$ :

a is associated with earlier onset of Alzheimer's disease

b is necessary for development of Alzheimer's disease

c is associated with increased risk of coronary heart disease

$\mathrm{d}$ is on chromosome 19

e can be used as a 'genetic prediction test' for Alzheimer's disease.

\begin{tabular}{|llllllllll|}
\hline \multicolumn{2}{|l}{ MCQ answers } & & & & & & \\
1 & & 2 & & 3 & & 4 & & 5 & \\
a & T & a & F & a & F & a & T & a & T \\
b & F & b & F & b & T & b & F & b & F \\
c & F & c & T & c & T & c & F & c & T \\
d & T & d & T & d & T & d & T & d & T \\
e & F & e & F & e & T & e & T & e & F \\
& & & & & & & & & \\
\hline
\end{tabular}

